\title{
Molecular dynamics study of the interaction between RNA-binding domain of NS1 influenza $A$ virus and various types of carbon nanotubes
}

\author{
Sara Az'hari*, Hamid Mosaddeghi and Yousef Ghayeb \\ Department of Chemistry, Isfahan University of Technology, Isfahan, 84156-83111, Iran
}

\begin{abstract}
Adsorption of biomolecules on the surface of carbon nanotubes (CNTs) is important in biological fields. Given the fact that RNA-binding domain (RBD) of NS1 protects the virus against antiviral materials, molecular dynamics simulation was employed to study the adsorption of RBD on the surface of CNTs. It was observed that RBD has a greater tendency to chiral CNTs. The results of $R_{g}$ (radius of gyration), root mean square fluctuation, hydrogen bonds, and secondary structure analysis indicate that the main chain of RBD is strongly stretched and CNT can function as a good adsorbent for RBD.
\end{abstract}

Keywords: Adsorption, carbon nanotubes, influenza A virus, molecular dynamics simulation, RNA-binding domain.

NANO-CARRIER drug delivery is nowadays being used to target molecules selectively ${ }^{1}$. Since the discovery of carbon nanotubes (CNTs) ${ }^{2}$, a growing interest has been shown by researchers to use them for many important goals due to their unique structure ${ }^{3}$ and advantageous properties. ${ }^{4}$ Many applications of nanotubes have been reported in biotechnological and biomedical fields ${ }^{5-7}$. CNTs display a major role as biocatalysts ${ }^{8,9}$, biosensors ${ }^{10-12}$ and biomedical devices ${ }^{13}$. The open-ended single-walled CNTs (SWCNTs) are rolled graphene sheets with carbon atoms and have a hollow cylindrical structure. These properties have encouraged use of SWCNTs as the best carriers. Both experimental and molecular simulation studies have focused on the investigation of CNTs as carriers of peptide ${ }^{14}, \mathrm{DNA}^{15}$, proteins $^{16}$ and drugs ${ }^{17,18}$. Some recent studies have also shown the effects of CNTs on natural biomolecular behaviour ${ }^{19,20}$. Although many experimental approaches have been employed to shed light on the interactions between biomolecules and CNT surfaces, the details of these interactions taking place at the molecular level are not understood. Moreover, the techniques developed so far cannot be used to explore the details about the functioning and conformation of many properties that depend on the non-covalent adsorption on CNTs. Adsorption process dynamics can yield a better under-

*For correspondence. (e-mail: s.azhari61@gmail.com) standing of environmental and biological activities of CNTs. Thus, molecular dynamics (MD) simulation is one of the most appropriate methods for accurate study of these processes at the atomic level.

Influenza A viruses are important pathogens with worldwide prevalence. Although their real host is waterfowl, they also infect humans ${ }^{21}$. In 1997, influenza A virus (H5N1) was transmitted from birds to humans in Hong Kong ${ }^{22}$. The highly virulent H5N1 and H3N2 subtypes of avian influenza viruses caused the disease to spread in China. The World Health Organization reported 105 mortalities among 186 human cases $^{23}$. It has been known that the wild outbreak of H5N1 subtype influenza A viruses in southeast Asia in these years are great candidates for causing the next flu pandemic. H5N1 and H3N2 viruses have been recognized for their great ability in human-to-human transmission ${ }^{24,25}$.

Clearly, these subtypes of influenza A virus are so virulent, with no certain reason yet found for it. On the other hand, certain contributing factors have been identified such as the influenza A virus-encoded non-structural (NS) protein 1 (ref. 26). The NS1 protein is a multifunctional protein that participates in both protein-protein and protein-RNA interactions. Two important domains have been explained in NS1 protein performing its multiple functions (Figure 1): the N-terminal structural domain (RBD) that protects the virus against antiviral materials,

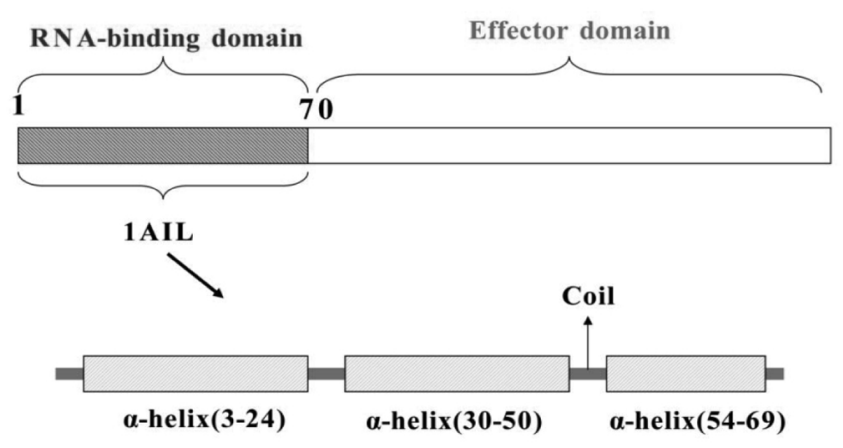

Figure 1. A simple plan of NS1 protein (of influenza A virus). RNAbinding domain consisting of 70 amino acids is known as 1AIL (PDB ID). This section of NS1 protein has three $\alpha$-helices and four coil sections.

CURRENT SCIENCE, VOL. 116, NO. 3, 10 FEBRUARY 2019 
and the C-terminal structural domain (effector domain) that prohibits the maturation and exportation of the host cellular antiviral mRNA.

The RBD domain is responsible for all RNA-binding activities of the full-length protein ${ }^{27}$. Because of the important role of RBD domain in the virulent power of NS1 protein, we selected this polypeptide for further studies. MD simulations were employed to study the effects of diameter and chirality of CNTs on the adsorption energies and the structural changes of RBD.

\section{Computational methods}

MD simulations were performed using the GROMACS software package ${ }^{28}$, with the OPLS-AA force field ${ }^{29}$. The structure file of the sightly protein, 1AIL (PDB ID) was selected $^{30}$. Water molecules and ions were removed from 1AIL structural file ${ }^{31}$. The VMD software was used for visualization and analysis of data, and to prepare the .pdb files for $\mathrm{CNTs}^{32}$. The GROMACS topologies input files for the CNTs were generated by $x 2$ top program. The parameters of uncapped CNTs are presented in Figure 2. Kaukonen et al. ${ }^{33}$ focused on the effect of diameter and chirality of CNTs on the Lennard-Jones (LJ) parameters by Density Functional Theory (DFT) method. They reported the values for LJ parameters of CNTs of all chiralities that are $\sigma_{\mathrm{cc}}=0.3534 \mathrm{~nm}, \varepsilon_{\mathrm{cc}}=0.2906 \mathrm{kj} \mathrm{mol}^{-1}$. These values are in good agreement with the recommended $\sigma, \varepsilon$ by Jorgensen and Schyman ${ }^{34}$. Therefore we used these values for the CNTs. The equilibrium values for structural parameters of bonded interactions (bond lengths and angles) were taken from the initially generated structure of CNTs, and the force constants for the bond and angle harmonic potentials were adopted from the OPLS-AA force field ${ }^{35}$. The length of CNTs was set at $4 \mathrm{~nm}$. All carbon atoms in the CNTs were uncharged
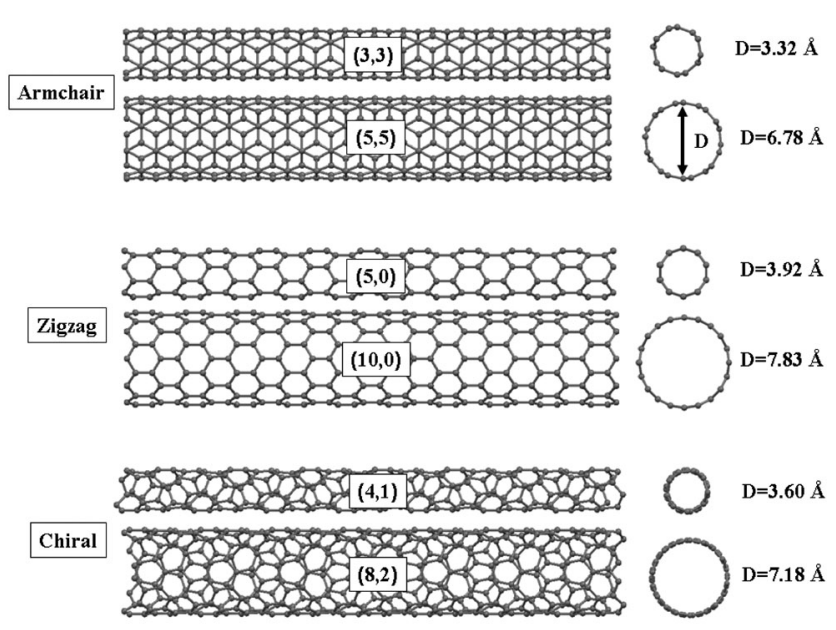

Figure 2. Types of CNTs and their parameters (D stands for diameter of each CNT). and flexible. To neutralize the systems, chloride ions were added to the boxes that contained RBD molecule.

The parameters of LJ potential for the cross-interactions between non-bonded atoms (e.g., CNT-protein, CNT-water) were obtained from the geometric combining rules ${ }^{36}$

$$
\begin{gathered}
\sigma_{i j}=\sqrt{\sigma_{i i} \sigma_{j j}}, \\
\varepsilon_{i j}=\sqrt{\varepsilon_{i i} \varepsilon_{j j}},
\end{gathered}
$$

where $i$ and $j$ indicate the type of particle. The size of simulation boxes was $6.75 \times 6.75 \times 6.75 \mathrm{~nm}^{3}$. Periodic boundary conditions were applied for all cases. Particle Mesh Ewald (PME) summation ${ }^{37}$ was used to calculate the long-ranged electrostatic interactions, with a cutoff distance of $12 \AA$. A time step of $1 \mathrm{fs}$ was set. TIP4P or TIP3P water models are commonly used in the OPLS simulations $^{38}$. The TIP3P water model was used in all simulations since it is a three-site model and significantly reduces the simulation costs. This water model is suitable for biomolecular simulations as well. There were 7970 water molecules in each simulation box to maintain a constant density of $1000 \mathrm{~kg} \mathrm{~m}^{-3}$. The pressure was held at 1 atm by Parrinello-Rahman coupling ${ }^{39}$ and the temperature was maintained at $300 \mathrm{~K}$ using the Berendsen thermostat $^{40}$. For computation of adsorption energy, simulation boxes consisting of the CNT-protein-water systems, the CNT-water systems, and the protein-water systems were built. The CNT-protein-water systems were run with starting structures that the closest atom to the surface of CNT was placed at $\sim 5 \AA$, to ensure sufficient space to contain several layers of water between protein and the CNT surface ${ }^{41,42}$.

For calculation of the energy of each system, 500,000step energy minimization was carried out at first. All systems underwent $1 \mathrm{~ns}$ MD simulation in NVT conditions and then $7 \mathrm{~ns}$ of MD runs in NPT ensemble, until the temperature, density and the total energy fluctuated around constant values. In all systems, we have not seen any significant changes in these parameters after $5 \mathrm{~ns}$ and they were equilibrated well.

The interaction energy, $E_{\text {int }}$, for all systems in MD simulations is defined as

$$
E_{\mathrm{int}}=E_{\mathrm{CNT}+\mathrm{Pro}}-E_{\mathrm{CNT}}-E_{\mathrm{Pro}}
$$

In eq. (3), $E_{\text {int }}$ stands for the total interaction between the protein (RBD) and the CNT surface, and $E_{\mathrm{CNT}+\text { pro, }}, E_{\mathrm{CNT}}$ and $E_{\text {Pro }}$ are the total energy of the CNT-protein complex in water, the total energy of CNT in water, and that of the protein in water respectively. These values were derived from the preserved frames by averaging the data for the last $1 \mathrm{~ns}$ (ref. 41). In general, 13 simulation cases were prepared, run and analysed. They are: RBD in 
water, six of them included a CNT in water (CNTs shown in Table 1) and the other cases consisting of RBD and CNT.

\section{Results and discussion}

\section{Effect of chirality and diameter of CNT on the adsorption energy}

The results show that the adsorption phenomenon occurred in all systems before $5 \mathrm{~ns}$. The mean values of adsorption energies are shown in Figure 3. It can be concluded that the RBD polypeptide has a greater tendency for adsorption on the surface of chiral CNTs. The least tendency was observed in the armchair CNTs. We observed a similar trend in our previous study ${ }^{43}$ in which we investigated the adsorption of twenty amino acids on the surface of CNTs with different chirality. There, we found that the chiral CNTs are the best and armchair CNTs are the worst type to capture the amino acids. This was because of the less aromaticity of the armchair CNTs. As known, adsorption is proportional to the surface of contact between two materials. Then we can expect that the adsorption of RBD on the surface of CNTs increased by increasing the diameter of CNTs. This point was only observed in each chirality type of CNTs. For example, the adsorption of RBD on the surface of $(3,3)$ CNT gave

Table 1. Secondary structure (SS) changes in $\mathrm{RBD}^{\mathrm{a}}$

\begin{tabular}{lll}
\hline ResID & SS of RBD in water & $\begin{array}{c}\text { SS of adsorbed RBD on } \\
\text { the surface of CNT }\end{array}$ \\
\hline 1,2 & $\mathrm{C}$ & $\mathrm{C}$ \\
$3-12$ & $\mathrm{H}$ & $\mathrm{H}$ \\
13 & $\mathrm{C}$ & $\mathrm{C}$ \\
$14-16$ & $\mathrm{H}$ & $\mathrm{H}$ \\
17 & $\mathrm{C}$ & $\mathrm{C}$ \\
18,19 & $\mathrm{H}$ & $\mathrm{H}$ \\
20 & $\mathrm{C}$ & $\mathrm{C}$ \\
$21-25$ & $\mathrm{H}$ & $\mathrm{H}$ \\
$26-29$ & $\mathrm{C}$ & $\mathrm{C}$ \\
$30-40$ & $\mathrm{H}$ & $\mathrm{H}$ \\
41 & $\mathrm{C}$ & $\mathrm{C}$ \\
$42-47$ & $\mathrm{H}$ & $\mathrm{H}$ \\
$48-50$ & $\mathrm{H}$ & $\mathrm{T}$ \\
$51-53$ & $\mathrm{C}$ & $\mathrm{C}$ \\
$54-58$ & $\mathrm{H}$ & $\mathrm{H}$ \\
59 & $\mathrm{C}$ & $\mathrm{C}$ \\
60 & $\mathrm{H}$ & $\mathrm{C}$ \\
61 & $\mathrm{H}$ & $\mathrm{H}$ \\
62 & $\mathrm{C}$ & $\mathrm{C}$ \\
$63-66$ & $\mathrm{H}$ & $\mathrm{H}$ \\
67 & $\mathrm{C}$ & $\mathrm{C}$ \\
$68-69$ & $\mathrm{H}$ & $\mathrm{T}$ \\
70 & $\mathrm{C}$ & $\mathrm{C}$ \\
\hline & &
\end{tabular}

${ }^{\mathrm{a}}$ The codes $\mathrm{C}, \mathrm{H}$ and $\mathrm{T}$ stand for the coil, $\alpha$-Helix and $\beta$-sheet component turn respectively. ResID indicates the sequence ID number of amino acids in RBD polypeptide.
$-235.6 \mathrm{kcal} \mathrm{mol}^{-1}$ energy. By doubling the diameter, this value increased to $-261.8 \mathrm{kcal} \mathrm{mol}^{-1}$ in $(5,5) \mathrm{CNT}$ case. But the adsorption of RBD on the surface of $(4,1) \mathrm{CNT}$ was more than the $(10,0) \mathrm{CNT}$ or $(5,5) \mathrm{CNT}$, although the diameter (and so the adsorbent surface) of $(4,1) \mathrm{CNT}$ was less than $(10,0)$ and $(5,5)$ cases. Maiti et al. ${ }^{44,45}$ showed that adsorption depends on the 'available surface area'. They investigated the number of contacts between the adsorbent and adsorbed materials. It appears that the adsorption is affected by the number of contacts. Then what is important in adsorption energy is available surface area and not only the surface of adsorbent.

In our case, it appears that the chirality of CNTs is affected by the available surface area. We can conclude that more aromaticity of chiral CNTs (for example $(4,1)$ $\mathrm{CNT}$ ) provides more available surface area for adsorption of RBD. Although $(4,1) \mathrm{CNT}$ has less diameter and less surface than $(5,5)$ or $(10,0)$ CNTs, it is a better adsorbent for RBD. This is because of more chirality, more aromaticity and more available surface area of $(4,1)$ CNT. Based on the adsorption energy values, it may be suggested that $(8,2) \mathrm{CNT}$ is the best case for adsorption of RBD.

\section{Conformational changes in $R B D$}

By tracking the trajectory files visually, we found that the structure of RBD had changed. In Figure 4, we can see the equilibrated structure of RBD near the $(4,1) \mathrm{CNT}$, before (Figure $4 a$ ) and after (Figure $4 b$ ) 8 ns MD simulation. Clearly, the structure of RBD is affected by CNT. It appears that the main chain of amino acids is opened and the whole of the RBD backbone is stretched. To gain more insight, we calculated the $R_{g}$ and RMSF parameters of RBD. Then we studied the changes in the secondary structure and intramolecular hydrogen bonds for RBD adsorbed on the surface of CNTs.

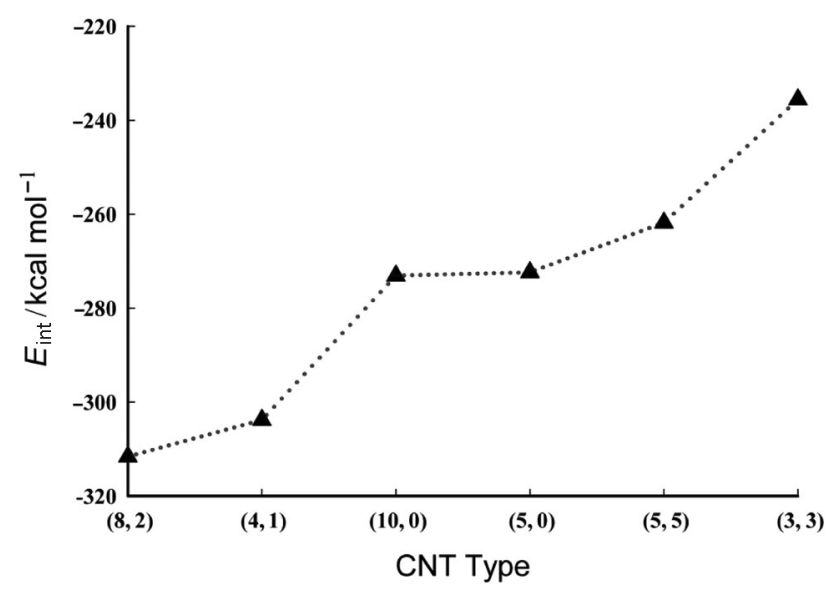

Figure 3. The mean values of adsorption energies (adsorption of RBD on the surface of CNTs).

CURRENT SCIENCE, VOL. 116, NO. 3, 10 FEBRUARY 2019 

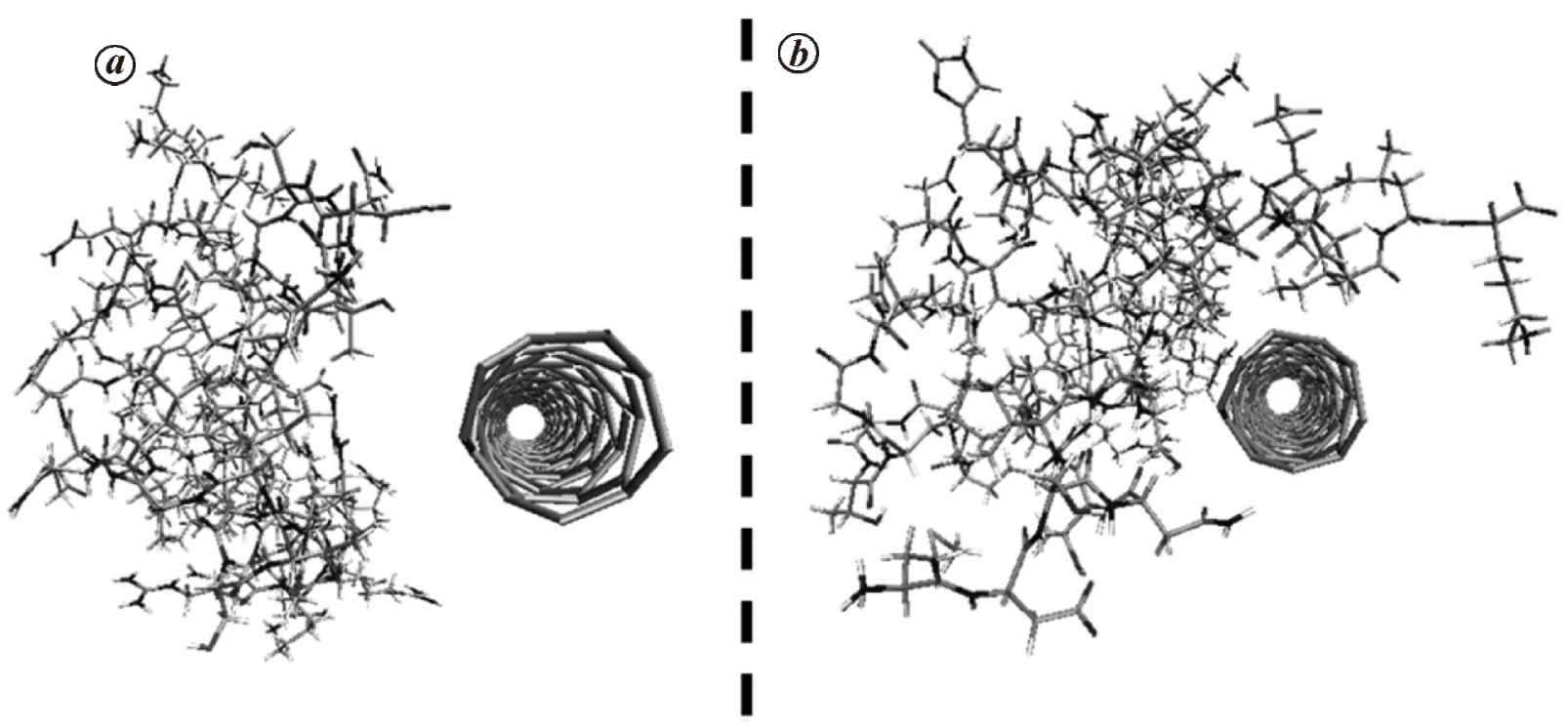

Figure 4. Equilibrated structure of RBD near the $(4,1)$ CNT. $\boldsymbol{a}$, before and $\boldsymbol{b}$, after adsorption.

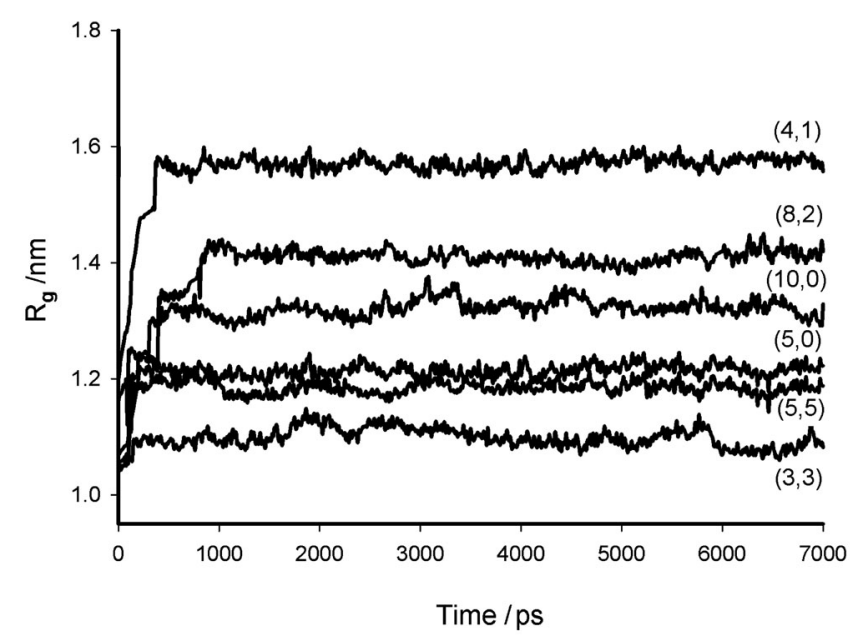

Figure 5. Values of $R_{g}$ versus MD time for adsorbed RBD on the surface of CNTs. (n, m) of each adsorbent CNTs are shown in graph.

\section{Investigation the $R_{g}$ of $R B D$}

In order to determine which of the CNTs has a greater effect on the structure of RBD, we calculated the $R_{g}$ parameter for RBD polypeptide. $R_{g}$ is defined as

$$
R_{g}=\sqrt{\frac{1}{N}\left\langle\sum_{i=1}^{N}\left(r_{i}-r_{\text {com }}\right)^{2}\right\rangle},
$$

where $r_{\mathrm{i}}$ and $r_{\text {com }}$ represent the position vector of each atom and the vector of centre-of-mass. If $R_{g}$ increases, it means that the structure is expanding. For RBD in pure water, the mean value of $R_{g}$ is calculated as $6.86 \pm$ $0.23 \AA$. The values of $R_{g}$ for adsorbed RBD on the surface of CNTs are shown in Figure 5.
Based on these data, the $R_{g}$ value of adsorbed RBD is greater than that in pure water. This indicates that the RBD structure is stretched due to the presence of CNTs. In other words, all CNTs (with any chirality and diameter) bring about RBD stretching. In addition, the chiral CNTs make the most changes in the structure of RBD. These results agree with the adsorption energy values obtained. The significant point is that the $(8,2) \mathrm{CNT}$ is the best adsorbent for RBD from the energy viewpoint, but the $(4,1)$ CNT is the best adsorbent for RBD from the structural changes viewpoint. The $R_{g}$ values of RBD that are adsorbed on the surface of CNTs are nearly two times with respect to pure water, which implies that the tertiary structure of RBD has obviously changed.

Therefore, we can conclude that chiral CNTs are generally the best candidate adsorbents of RBD. It may be claimed that the $(8,2) \mathrm{CNT}$ (the chiral CNT with larger diameter) is the best collector adsorbent for RBD from a viral medium. Where the changes of RBD structure and its biological functions by a CNT are sought, the $(4,1)$ CNT (the chiral CNT with the smaller diameter) seems to be a better choice.

\section{Investigation the RMSF parameter of $R B D$ residues}

We then calculated the RMSF of RBD adsorbed on the surface of CNTs and that of RBD in pure water. In Figure 6 , RMSF values of adsorbed RBD are plotted versus the number of RBD residues. For comparison, the RMSF values of RBD in pure water are shown. As seen, most changes in the RMSF values (compared to any RBD residues in pure water) belonged to Lys 70, Ile 68, Leu 69, Asp 2 and Met 1. These data show that the adsorption of RBD on the surface of CNTs does not lead to any 

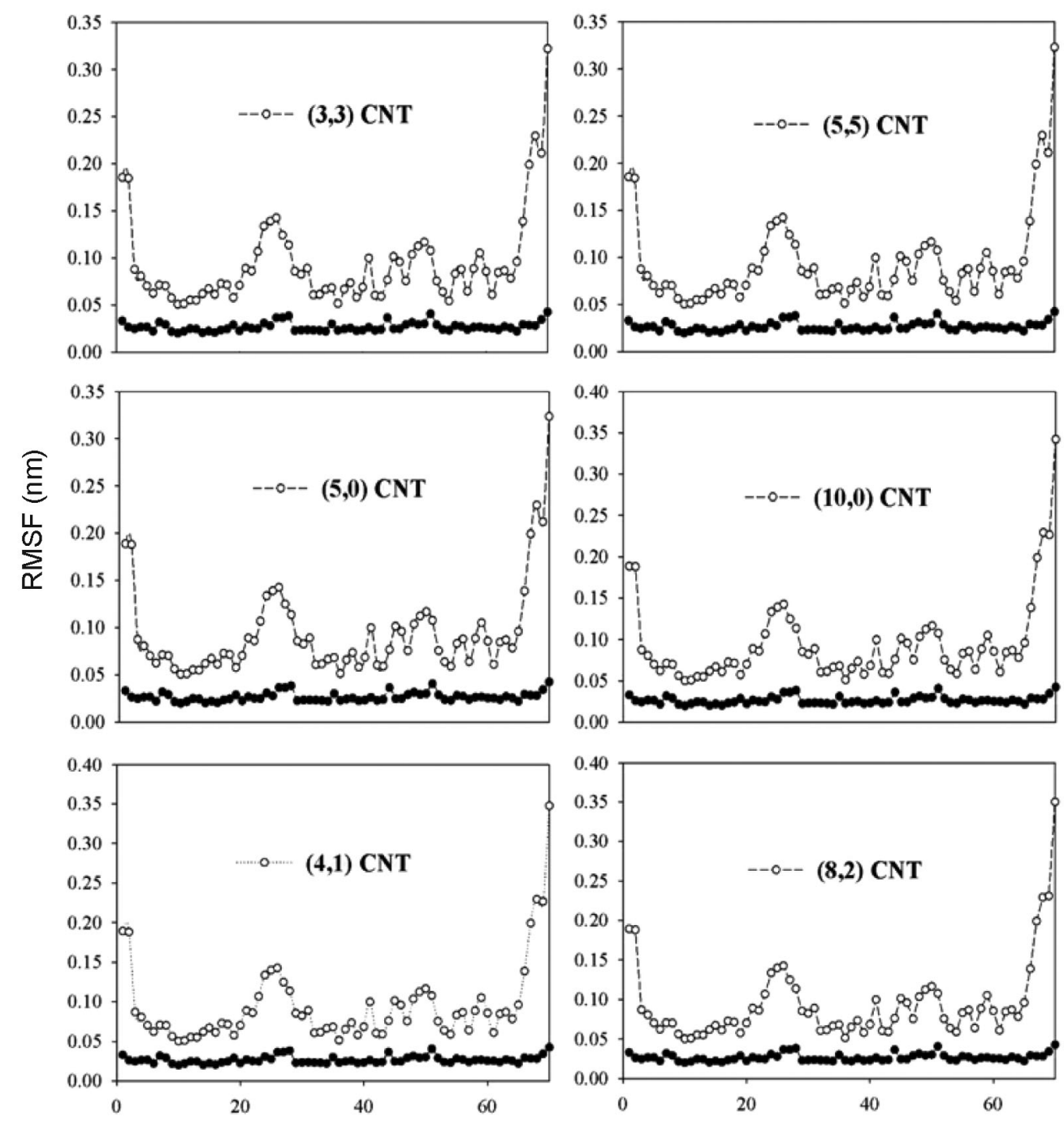

Residue number

Figure 6. RMSF values of 70 RBD residues before and after adsorption on the surface of CNTs shown as black and white circles respectively. Each CNT type is presented with $(n, m)$ in the graphs.

significant structural changes. Because the greatest change in RMSF values in all systems is observed for terminal amino acids, we can conclude that in RBD structure, both $\mathrm{N}$-terminal and $\mathrm{C}$-terminal amino acids are affected by CNTs. According to RMSF results, it appears that RBD adsorption does not greatly change its secondary structure and that only the main chain of RBD is stretched. To get more details about the structural changes of RBD, we focused on the secondary structure analysis.

\section{Secondary structural changes in $R B D$}

Table 1 lists the initial and final results of the secondary structure of RBD evaluated by VMD sequence viewer. The interesting point is that all types of CNTs (chiral, zigzag and armchair) with any diameter value, cause similar changes in the secondary structure of RBD. Table 1 shows that the dominant structure of RBD in pure water is a $\alpha$-helix. After adsorption, $\alpha$-helix is the dominant structure of RBD as well. Only the five amino acids (Ser 44, Thr 49, Leu 50, Ile 68 and Leu 69) that have participated in $\alpha$-helices transform into $\beta$-sheet component turns and Val 60 (with $\alpha$-helix structure in initial form) transforms to random coil structure.

According to these data, it can be concluded that the secondary structure of RBD has no significant changes. But it appears that the tertiary structure has changed, because of the transformation of $\alpha$-helix to $\beta$-sheet. We know that the change in tertiary structure of a protein alters the number of intramolecular $\mathrm{HBs}^{44}$. Therefore, we investigated the intramolecular HBs of RBD before and after adsorption. 
RESEARCH ARTICLES

Table 2. Number and lifetime values of intramolecular HBs for RBD

\begin{tabular}{lccccccc}
\hline Adsorbent CNT (n, m) & - & $(8,2)$ & $(4,1)$ & $(10,0)$ & $(5,0)$ & $(5,5)$ & $(3,3)$ \\
\hline Number of intramolecular HBs in RBD & 51.789 & 55.423 & 55.937 & 55.024 & 53.820 & 53.140 & 52.148 \\
Mean value of HBs lifetime (ps) & 0.44 & 0.58 & 0.61 & 0.56 & 0.51 & 0.47 & 0.47 \\
\hline
\end{tabular}

\section{Effect of CNT on the intramolecular hydrogen bonds in $R B D$}

There are some intramolecular HBs in all biomacromolecules that influence the properties of proteins and their biological functions ${ }^{46}$. We calculated the number and lifetime of intramolecular HBs for RBD in all systems, using the GROMACS package. Optimal cutoff distance was determined as $3.5 \AA$ and optimal cutoff angle was set at $30^{\circ}$. The results are shown in Table 2.

These data indicate that the adsorption of RBD on the surface of CNTs causes increase in the number and lifetime of intramolecular HBs. The CNT which creates the most $R_{g}$ value in RBD molecule forms the most intramolecular HBs. Where the number of intramolecular HBs increases, the lifetime of these HBs is more. It appears that when the folded main chain of polypeptide was opened and the tertiary structure of protein was changed, more intramolecular HBs with more durability were formed to increase the stability of protein structure. The intramolecular $\mathrm{HB}$ properties agree with the results of secondary structural changes. The adsorption of RBD on the surface of CNTs changes the $\alpha$-helices (in two domains) to the $\beta$-sheet component turns. It is known that when every backbone $\mathrm{N}-\mathrm{H}$ group donates a $\mathrm{HB}$ to the backbone $\mathrm{C}=\mathrm{O}$ group of four residues earlier amino acids (i+ $4 \rightarrow \mathrm{i}$ ) hydrogen bonding, an $\alpha$-helix is formed ${ }^{47}$. However, formation of a $\beta$-sheet component turn is due to $(\mathrm{i}+1 \rightarrow \mathrm{i})$ or $(\mathrm{i}+2 \rightarrow \mathrm{i})$ hydrogen bonding ${ }^{48,49}$. Therefore it is certainly acceptable that when an $\alpha$-helix transforms into a $\beta$-sheet component turn, the number of intramolecular HBs be increased.

\section{Conclusions}

The adsorption process of RBD on the surface of CNTs with different chirality and diameters was investigated by MD simulation. Adsorption occurred in all systems before $5 \mathrm{~ns}$. The RBD polypeptide has a greater tendency to be adsorbed on the surface of chiral CNTs. Increasing the diameter of CNT leads to greater adsorption energy. Visual analysis showed that the structure of RBD is affected by CNT. It appears that the main chain of amino acids is opened and the whole RBD backbone is stretched. To gain more insight on this process, we investigated the conformational changes of RBD during the adsorption. The values of $R_{g}$ for adsorbed RBD have been increased by the presence of CNT. The tertiary structure of RBD has obviously changed and chiral CNTs lead to the most changes. The significant point is that the $(8,2) \mathrm{CNT}$ is the best adsorbent for RBD from the energy viewpoint, and the $(4,1)$ CNT is the best adsorbent for RBD from the structural changes viewpoint. RMSF analyses show that both N-terminal and C-terminal amino acids of RBD (Lys 70, Ile 68, Leu 69, Asp 2 and Met 1) are affected by CNT. But it does not greatly change the secondary structure of RBD. We then focused on the secondary structure analysis. During adsorption, only five amino acids (Ser 44, Thr 49, Leu 50, Ile 68 and Leu 69) transform from $\alpha$ helices to $\beta$-sheet component turns and Val 60 transforms from $\alpha$-helix to random coil structure. Calculation of the intramolecular HBs in RBD molecule indicates that the HB's number and lifetime have increased after adsorption. These data are in agreement with the results of secondary structure changes. Finally, we conclude that CNTs, especially chiral type, can be used as an adsorbent for influenza A virus from a viral medium.

1. Mansoori, G. A., Mohazzabi, P., Mccormack, P. and Jabbari, S., Nanotechnology in cancer prevention, detection and treatment: bright future lies ahead. WRSTSD, 2007, 4, 226-257.

2. Iijima, S., Helical microtubules of graphitic carbon. Nature, 1991, 354, 56-58.

3. Kam, N. W. S., Jessop, T. C., Wender, P. A. and Dai, H., Nanotube molecular transporters: internalization of carbon nanotubeprotein conjugates into mammalian cells. J. Am. Chem. Soc., 2004, 126, 6850-6851.

4. Lin, Y. et al., Advances toward bioapplications of carbon nanotubes. J. Mater. Chem., 2004, 14, 527-541.

5. Mandal, H. S., Su, Z., Ward, A. and Tang, X., Carbon nanotube thin film biosensors for sensitive and reproducible whole virus detection. Theranostics, 2012, 2, 251-257.

6. Boero, C., Carrara, S., Vecchio, G. D., Calza, V. and Micheli, G. D., Targeting of multiple metabolites in neural cells monitored by using protein-based carbon nanotubes. Sensor. Actuate. B. Chem., 2011, 157, 216-224.

7. Jana, A. K. and Sengupta, N., Surface induced collapse of A $\beta 1-42$ with the F19A replacement following adsorption on a single walled carbon nanotube. Biophys. Chem., 2013, 184, 108-115.

8. Mitchell, D. T., Lee, S. B., Trofin, L., Li, N., Nevanen, T. K., Soderlund, H. and Martin, C. R., Smart nanotubes for bioseparations and biocatalysis. J. Am. Chem. Soc., 2002, 124, 1186411865 .

9. Bi, Y. H., Huang, Z. L. and Zhao, Y. D., Interactions of cytochrome c with DNA at glassy carbon surface. Biophys. Chem., 2005, 116, 193-198.

10. Azamian, B. R., Davis, J. J., Coleman, K. S., Bagshaw, C. B. and Green, M. L., Bioelectrochemical single-walled carbon nanotubes. J. Am. Chem. Soc., 2002, 124, 12664-12665.

11. Veetil, J. V. and Ye, K., Development of immunosensors using carbon nanotubes. Biotechnol. Prog., 2007, 23, 517-531. 


\section{RESEARCH ARTICLES}

12. Vatsyayan, P., Bordoloi, S. and Goswami, P., Large catalase based bioelectrode for biosensor application. Biophys. Chem., 2010, 153, 36-42.

13. Kam, N. W. S., O'Connell, M., Wisdom, J. A. and Dai, H., Carbon nanotubes as multifunctional biological transporters and nearinfrared agents for selective cancer cell destruction. Proc. Natl. Acad. Sci. USA, 2005, 102, 11600-11605.

14. Kang, Y., Wang, Q., Liu, Y. C., Wu, T., Chen, Q. and Guan, W. J., Dynamic mechanism of collagen-like peptide encapsulated into carbon nanotubes. J. Phys. Chem. B., 2008, 112, 4801-4807.

15. Pei, Q. X., Lim, C. G., Cheng, Y. and Gao, H., Molecular dynamics study on DNA oligonucleotide translocation through carbon nanotubes. J. Chem. Phys., 2008, 129, 125101-125108.

16. Tsang, S. C., Davis, J. J., Green, M. L. H., Hill, H. A. O., Leung, Y. C. and Sadler, P. J., Immobilization of small proteins in carbon nanotubes: high-resolution transmission electron microscopy study and catalytic activity. J. Chem. Soc. Chem. Commun., 1995, 17, 2579-2580.

17. Hilder, T. A. and Hill, J. M., Probability of encapsulation of paclitaxel and doxorubicin into carbon nanotubes. Micro. Nano. Lett., 2008, 3, 41-49.

18. Chen, Q., Wang, Q., Liu, Y. C., Wu, T., Kang, Y., Moore, J. D. and Gubbins, K. G., Energetics investigation on encapsulation of protein/peptide drugs in carbon nanotubes. J. Chem. Phys., 2009, 131, 015101-015106.

19. Chen, B. D., Yang, C. L., Yang, J. S., Wang, M. S. and Ma, X. G., Dynamic mechanism of HIV replication inhibitor peptide encapsulated into carbon nanotubes. Curr. Appl. Phys., 2013, 13, 10011007.

20. Fu, Z. M., Luo, Y., Derreumaux, P. and Wei, G. H., Induced $\beta$ barrel formation of the Alzheimer's A $\beta 25-35$ oligomers on carbon nanotube surfaces: implication for amyloid fibril inhibition. Biophys. J., 2009, 97, 1795-1803.

21. Webster, R. G., Bean, W. J., Gorman, O. T., Chambers, T. M. and Kawaoka, Y., Evolution and ecology of influenza A viruses. Microbiol. Rev., 1992, 56, 152-179.

22. Seo, S. H., Hoffmann, E. and Webster, R. G., Lethal H5N1 influenza viruses escape host anti-viral cytokine responses. Nat. Med., 2002, 8, 950-954.

23. Lu, P. S., Early diagnosis of avian influenza. Science, 2006, 312, 337.

24. Horimoto, T. and Kawaoka, Y., Influenza: lessons from past pandemics, warnings from current incidents. Nat. Rev. Microbiol., 2005, 3, 591-600.

25. Noah, D. L. and Krug, R. M., Influenza virus virulence and its molecular determinants. Adv. Virus. Res., 2005, 65, 121-145.

26. Solorzano, A., Webby, R. J., Lager, K. M., Janke, B. H., GarciaSastre, A. and Richt, J. A., Mutations in the NS1 protein of swine influenza virus impair anti-interferon activity and confer attenuation in pigs. $J$. Virol., 2005, 79, 7535-7543.

27. Qian, X. Y., Lu, Y., Montelione, G. T. and Krug, R. M., An amino-terminal polypeptide fragment of the influenza virus NS1 protein possesses specific RNA-binding activity and largely helical backbone structure. $R N A, 1995,1,948-956$.

28. Van der Spoel, D., Lindahl, E., Hess, B., Groenhof, G., Mark, A. E. and Berendsen, H. J. C., GROMACS: fast, flexible, and free. J. Comput. Chem., 2005, 26, 1701-1718.

29. Jorgensen, W. L., Maxwell, D. S. and Tirado-Rives, J., Development and testing of the OPLS all-atom force field on conformational energetics and properties of organic liquids. J. Am. Chem. Soc., 1996, 118, 11225-11236.

30. Liu, J., Lynch, P. A., Chien, C. Y., Montelione, G. T., Krug, R. M. and Berman, H. M., Crystal structure of the unique RNA-binding domain of the influenza virus NS1 protein. Nat. Struct. Mol. Biol., 1997, 4, 896-899.
31. http://www.ks.uiuc.edu/Training/Tutorials/science/membrane/memtutorial.pdf

32. Humphrey, W., Dalke, A. and Schulten, K., VMD: Visual molecular dynamics. J. Mol. Graph., 1996, 14, 33-38.

33. Kaukonen, M., Gulans, A., Havu, P. and Kauppinen, E., LennardJones parameters for small diameter carbon nanotubes and water for molecular mechanics simulations from van der Waals density functional calculations. J. Comput. Chem., 2012, 33, 652.

34. Jorgensen, W. L. and Schyman, P., Exploring adsorption of water and ions on carbon surfaces using a polarizable force field. J. Phys. Chem. Lett., 2013, 4, 468.

35. Jorgensen, W. L. and Severance, D. L., Aromatic-aromatic interactions: free energy profiles for the benzene dimer in water, chloroform, and liquid benzene. J. Am. Chem. Soc., 1990, 112, 4768.

36. Jorgensen, W. L., Maxwell, D. S. and Tirado-Rives, J., Development and testing of the OPLS all-atom force field on conformational energetics and properties of organic liquids. J. Am. Chem. Soc., 1996, 118, 11225.

37. Darden, T., York, D. and Pedersen, L., Particle mesh Ewald: An $N \cdot \log (N)$ method for Ewald sums in large systems. J. Chem. Phys., 1993, 98, 10089-10092.

38. Van der Spoel, D. and Lindahl, E., Brute-force molecular dynamics simulations of Villin headpiece: comparison with NMR parameters. J. Phys. Chem. B., 2003, 107, 11178-11187.

39. Parrinello, M. and Rahman, A., Polymorphic transitions in single crystals: A new molecular dynamics method. J. Appl. Phys., 1981, 52, 7182-7190.

40. Berendsen, H. J. C., Postma, J. P. M., van Gunsteren, W. F., DiNola, A. and Haak, J. R., Molecular-dynamics with coupling to an external bath. J. Chem. Phys., 1984, 81, 3684-3690.

41. Shen, J. W., Wu, T., Wang, Q. and Kang, Y., Induced stepwise conformational change of human serum albumin on carbon nanotube surfaces. Biomaterials, 2008, 29, 3847-3855.

42. Mosaddeghi, H., Alavi, S., Kowsari, M. H. and Najafi, B., Simulations of structural and dynamic anisotropy in nano-confined water between parallel graphite plates. J. Chem. Phys., 2012, 137, 184703-184712.

43. Az'hari, S. and Ghayeb, Y., Effect of chirality, length and diameter of carbon nanotubes on the adsorption of 20 amino acids: a molecular dynamics simulation study. Mol. Simulat., 2014, 40, 392-398.

44. Santosh, M., Panigrahi, S., Bhattacharyya, D., Sood, A. K. and Maiti, P. K., Unzipping and binding of small interfering RNA with single walled carbon nanotube: a platform for small interfering RNA delivery. J. Chem. Phys., 2012, 136, 065106.

45. Vasumathi, V., Pramanik, D., Sood, A. K. and Maiti, P. K., Structure of a carbon nanotube-dendrimer composite. Soft Matter., 2013, 9, 1372-1380.

46. Feher, J., Quantitative Human Physiology, Academic Press, Massachusetts, 2012, pp. 100-109.

47. Chamberlain, A. K. and Bowie, J. U., Evaluation of C-H...O hydrogen bonds in native and misfolded proteins. J. Mol. Biol., 2002, 322, 497-503.

48. Pauling, L., Corey, R. B. and Branson, H. R., The structure of proteins: two hydrogen-bonded helical configurations of the polypeptide chain. Proc. Natl. Acad. Sci. USA, 1957, 37, 205-211.

49. Toniolo, C. and Benedetti, E., Intramolecularly hydrogen-bonded peptide conformation. CRC. Crit. Rev. Biochem., 1980, 9, 1-44.

ACKNOWLEDGEMENT. Financial support by the Research Council of Isfahan University of Technology is gratefully acknowledged.

Received 19 March 2015; revised accepted 3 November 2018

doi: $10.18520 / \mathrm{cs} / \mathrm{v} 116 / \mathrm{i3} / 398-404$ 